\title{
Ensemble-based optimization of interwell connectivity in heterogeneous waterflooding reservoirs
}

\author{
Daigang Wang a, b, *, Yong Li ${ }^{\text {a }}$, Bailian Chen ${ }^{c,{ }^{* *}}$, Yongle $\mathrm{Hu}^{\mathrm{a}}$, Baozhu Li ${ }^{\mathrm{a}}$, Dapeng Gao ${ }^{\mathrm{d}}$, \\ Bing $\mathrm{Fu}^{\mathrm{e}}$ \\ a Research Institute of Petroleum Exploration \& Development, PetroChina, Beijing 100083, China \\ ${ }^{\mathrm{b}}$ School of Earth and Space Sciences, Peking University, Beijing 100871, China \\ ${ }^{\mathrm{c}}$ McDougall School of Petroleum Engineering, University of Tulsa, United States \\ ${ }^{\mathrm{d}}$ Institute of Mechanics, Chinese Academy of Sciences, Beijing 100190, China \\ e College of Petroleum Engineering, China University of Petroleum-Beijing (CUPB), Beijing 102249, China
}

\section{A R T I C L E I N F O}

\section{Article history:}

Received 27 September 2016

Received in revised form 20 November 2016

Accepted 20 December 2016

Available online 29 December 2016

\section{Keywords:}

Capacitance-resistance model

Koval fractional-flow equation

Interwell connectivity

Ensemble-based optimization technique

Hybrid nonlinear constraint

\begin{abstract}
A B S T R A C T
Estimation of interwell connectivity is of great importance to optimization of injection-production scheme and decision-making of potential-tapping strategies during the later stage of waterflooding. However, the traditional reservoir simulation requires detailed information of various reservoir/fluid parameters, which is time-consuming and difficult to obtain the reliable estimates due to large uncertainties. The capacitance-resistance model inferred from field injection and production data provides an attractive alternative to understanding the interwell connectivity relationship and close-loop reservoir management. For this study, the producer-based and injector-producer pair-based capacitance resistance model, CRMP and CRMIP, are employed to compute liquid production rate of each producer, respectively, followed by description of observed water cut data using the Koval fractional-flow equation. Then, this paper proposes a novel framework that enables the newly developed Stochastic Simplex Appropximate Gradient (StoSAG) algorithm to optimize interwell connectivity in waterflooding reservoirs by preconditioning the hybrid nonlinear constraints, which is further validated by a heterogeneous synthetic case. The results show that, compared to the projected-gradient (PG) and EnKF methods, the StoSAG optimization technique can handle the sequential data assimilation in large-scale nonlinear dynamics more robustly; due to more degrees of freedom, the CRMIP representation captures the reservoir's dynamic behavior better than CRMP, resulting in a more satisfactory estimation of geological parameters relative to each reservoir control volume; The Koval fractional-flow equation are effective to represent the water-producing characteristics from small-to-large water cut period, but a great deviation will be caused during the extra-high water cut stage $\left(f_{\mathrm{w}}>90 \%\right)$ because of its inherent drawbacks.
\end{abstract}

๑) 2016 Elsevier B.V. All rights reserved.

\section{Introduction}

Estimation of interwell connectivity is essential to field development project design, which is also of great importance to optimization of injection-production scheme, analysis of remaining oil distribution and decision-making of potential-tapping strategies. However, the traditional reservoir simulation is usually based on

\footnotetext{
* Corresponding author.Research Institute of Petroleum Exploration \& Development, PetroChina, Beijing 100083, China.

** Corresponding author.

E-mail addresses: dgwang@pku.edu.cn (D. Wang), bailian-chen@utulsa.edu (B. Chen).
}

the finite difference method, which requires detailed information of various reservoir/fluid parameters, such as porosity, permeability, relative permeability and saturation in grid blocks. Since information about those parameters is also limited by measuring techniques, vast majority of those parameters are mainly determined by interpolation from that of well points, usually leading to a great deviation. Furthermore, uncertainties of the reservoir/fluid parameters used for history matching field production data are further intensified by the inherent drawbacks of the existing measuring techniques, which cannot meet the requirement of good physical understanding to reservoir (Jin et al., 2004; Kang et al., 2012; Zhao et al., 2015a). Using historical data of injection and production rates has proven to be an attractive alternative to 
accurate estimation of interwell connectivity in water flooded reservoirs. The simplified reservoir models proposed mainly consist of the linear multivariate regression model (Gentil, 2005), the capacitance-resistance model (CRM) (Yousef et al., 2006a, 2006b; Sayarpour et al., 2007; Moreno, 2013; Mamghaderi and Pourafshary, 2013; Zhang et al., 2015; Holanda et al., 2015), the multiwell productivity index (Kaviani et al., 2010), the flownetwork model (Lerlertpakdee et al., 2014) and the interwell numerical simulation model (Zhao et al., 2015b, 2016). As an alternative to reservoir simulation, the CRM representation is generally based on signal-processing techniques and a total mass balance equation with compressibility, in which injection rate are assumed as input signals and production rates are treated as output signals of the integrated dynamic system, respectively, and characterizes a flooded reservoir by rapid estimating connectivity coefficients, time constants and productivity indices using only the producers' bottom-hole pressure if necessary and production/injection rate for history matching. Due to its high computational efficiency and capability for reservoir performance prediction, the CRM has gained substantial popularity to close-loop reservoir management and production optimization, especially in areas of primary recovery (Nguyen, 2012), waterflooding (Lee et al., 2011; Tafti et al., 2013), gas flooding (Nguyen, 2012; Sakazar-Bustamante et al., 2012) and $\mathrm{CO}_{2}$ geological sequestration (Tao and Bryant, 2015).

However, all the above-mentioned simplified reservoir models are single-phase flow model merely history matching and predicting the liquid production rate, and not capable of separating the oil production from the total production adaptively, which bring about plenty of difficulties for decision-making of potential-tapping strategies during the later stage of waterflooding. To tackle the deficiencies, Gentil et al. (Gentil, 2005) present an empirical linear relationship between the natural logs of instantaneous water-oil ratio and cumulative water injection, which is usually valid in mature water floods. For the same reason, it provides often a good approximation at the late life of a waterflood when water cut is large. Zhao et al. (2015a; 2015b; 2016) assume that the rock and fluid are incompressible during the tracking process of water cut data. Instead of solving the saturation distribution along each onedimensional space, the Buckley-Leverett waterflood front equation is applied to compute water cut at the downstream well of each well pair, which keeps a fast computation speed. Nevertheless, the Buckley-Leverett model has many assumptions such as homogenous media, one dimension flow, incompressible system, negligible gravity and capillarity, all of which should be carefully understood prior to application. Cao et al. $(2014,2015)$ provide a water fractional flow equation inferred from field production data using the Koval theory. By history matching field water cut data, two model parameter, Koval factor and drained pore volume, will be estimated. The Koval fractional-flow equation is far more general and flexible because there is no specific assumption regarding the immiscible displacement, which has the advantage to address the issue of viscous fingering in a miscible displacement. Furthermore, due to the high complexity of flooded reservoirs and urgent requirement of real-time production optimization, the gradient-based methods (e.g., the steepest-descent method, the projected-gradient method, and so on) commonly used to solve optimization problems where the direction of search for a local minimum is obtained by computing the gradient of objective function with respect to the geological parameters such as connectivity coefficients, time constants and productivity indices, will not be suitable in many cases, particularly, the large-scale heterogeneous waterflood reservoirs with hybrid nonlinear constraints. Nowadays, the derivative-free optimization techniques have garnered attentions in the computational mathematics literature. Whereby, the ensemble-based methods have proven to be one of the most popular derivative- free optimization techniques for many applications due to its versatility and simplicity, involving in close-loop reservoir management (Chen et al., 2009, 2012; Do and Reynolds, 2013; Zhao et al., 2013), estimation of optimal well controls (Su and Oliver, 2010; Oliveira and Reynolds, 2014; Fonseca et al., 2014), and EOR screening (Odi et al., 2010; Katterbauer, 2015; Chen and Reynolds, 2016). However, there are few proposals for interwell connectivity estimation with the ensemble-based optimization techniques (Zhang et al., 2015; Jafroodi and Zhang, 2011).

Here, we develop a novel framework that enables the newly developed Stochastic Simplex Appropximate Gradient (StoSAG) algorithm to optimize interwell connectivity in heterogeneous waterflooding reservoirs by preconditioning the hybrid nonlinear constraints. This paper is organized as follows: First, we provide the formulation and architecture of different capacitance-resistance models and Koval fractional flow equation, respectively, followed by a brief description of the ensemble-based optimization process for solving hybrid nonlinear constrained problems. Thereafter, with respect to a heterogeneous synthetic case, the proposed technique is performed to history match the observed production data adaptively, and thus determine the interwell geological parameters such as connectivity coefficients, time constants, and drained pore volumes. Finally, we summarize the results and present the conclusions of this work.

\section{Methodology}

In this section, the producer-based and injector-producer pairbased capacitance resistance models (Sayarpour et al., 2007), CRMP and CRMIP, are respectively introduced to compute liquid production rate of each producer within a specific reservoir control volume in terms of considering the impact of interwell connectivity, time lag and reservoir/fluid compressibility. Moreover, the Koval water fractional-flow equation developed by Cao et al. (2014, 2015) is applied to separate the oil production from total production. Thereafter, a novel ensemble-based optimization framework preconditioning hybrid nonlinear constraints is then provided to minimize the squared difference between the predicted and observed production data, so that the interwell geological parameters such as connectivity coefficients, time constants, and drained pore volumes will be eventually estimated.

\subsection{Capacitance-resistance model}

\section{(1) Producer-based representation-CRMP}

As shown in Fig. 1, the producer-based representation (CRMP) divides the reservoir into a series of control volumes based each producer and includes all the injectors that influence their production rates, which may be all injectors, unless some extra

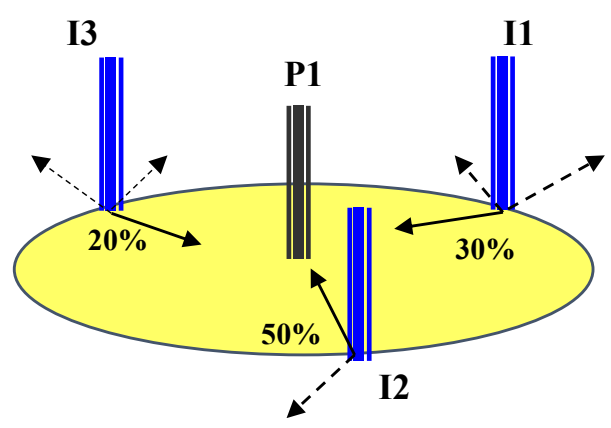

Fig. 1. Schematic of reservoir control volume in the CRMP representation. 
constraints are imposed. The CRMP model considers one time constant for each producer and one connectivity coefficient for each injector-producer pair, therefore, the continuity equation (Sayarpour et al., 2007) for producer $j$ is as follows.

$\frac{d q_{j}(t)}{d t}+\frac{1}{\tau_{j}} q_{j}(t)=\frac{1}{\tau_{j}} \sum_{i=1}^{N_{i n j}} f_{i j} I_{i}(t)-J_{j} \frac{d P_{w f, j}}{d t}$

where $q_{j}(t)$ is the liquid production rate of producer $j$ at time $t, \mathrm{~m}^{3} /$ $\mathrm{d}, j=1,2, \ldots, N_{\text {pro }} ; I_{i}(t)$ is the injection rate of injector $i$ at time $t$, $\mathrm{m}^{3} / \mathrm{d}, i=1,2, \cdots, N_{i n j} ; N_{\text {pro }}$ and $N_{i n j}$ are the total number of producers and injectors, respectively; $P_{w f, j}$ is the bottom-hole pressure of producer $j$ at time $t, \mathrm{MPa} ; J_{j}$ is the productivity index of producer $j, \mathrm{~m}^{3} /(\mathrm{MPa} \cdot \mathrm{d}) ; \tau_{j}$ is the time constant for volume drained by producer $j$, which is a measure of the time necessary for pressure wave to propagate in porous media and effectively influence the production signal, $\tau=C_{t} \cdot V_{p} / J$, The total compressibility $c_{t}$, the drained pore volume $V_{p}$ and the productivity index $J$ play an integrated impact on the time constant; $f_{i j}$ is the connectivity between injector (i) and producer $(j), f_{i j} \in[0,1]$, the closer the $f_{i j}$ approaches to zero, the poorer the connectivity between $i-j$ pair.

For any time interval $t_{k}$, when changes of bottom-hole pressure are considered, the semi-analytical solution to Eq. (1) is derived using time and space superposition, which is given by (Sayarpour et al., 2007)

$$
\begin{aligned}
q_{j}\left(t_{k}\right)= & q_{j}\left(t_{0}\right) e^{-\left(\frac{t_{k}-t_{0}}{\tau_{j}}\right)}+\sum_{s=1}^{k}\left\{e^{-\left(\frac{t_{k}-t_{s}}{\tau_{j}}\right)}\left(1-e^{-\frac{\Delta t_{s}}{\tau_{j}}}\right)\right. \\
& {\left.\left[\sum_{i=1}^{N_{i n j}}\left[f_{i j} \cdot I_{i}^{(s)}\right]-J_{j} \cdot \tau_{j} \frac{\Delta p_{w f, j}^{(s)}}{\Delta t_{s}}\right]\right\} }
\end{aligned}
$$

where the superscript $s$ is index of time interval; $\Delta t_{s}$ is the $s$ th time interval, $\mathrm{d} ; q_{j}\left(t_{k}\right)$ is the liquid production rate of producer $j$ at time $t_{k}, \mathrm{~m}^{3} / \mathrm{d} ; q_{j}\left(t_{0}\right)$ is the liquid production rate of producer $j$ at the initial time $t_{0}$ of history matching, $\mathrm{m}^{3} / \mathrm{d} ; I_{i}^{(s)}$ and $\Delta p_{w f . j}^{(s)}$ are the rate of injector $i$ and changes in bottom hole pressure of producer $j$ during time interval $t_{s-1}$ to $t_{s}$, respectively.

To match the production history for a pattern of $N_{i n j}$ injectors and $N_{\text {pro }}$ producers by the CRMP representation as shown in Eq. (2), the CRMP has $\left(N_{i n j}+3\right)$ unknown model parameters for each producer $j: f_{i j}\left(i=1,2, \ldots, N_{i n j}, j=1,2, \ldots, N_{p r o}\right), q_{j}\left(t_{0}\right), \tau_{j}$ and $J_{j}$. Therefore, to use the CRMP in a field, one must evaluate $N_{\text {pro }}\left(N_{i n j}+\right.$ 3 ) model unknowns. Nevertheless, pressure data are often not available while rate data are of great abundance. If the bottom-hole pressure at the producers is kept constant, the model parameters decreases to $N_{\text {pro }}\left(N_{i n j}+2\right)$ for the CRMP.

Fig. 2 shows the influence of connectivity coefficient and time constant for a CRMP production rate response with only one injector and one producer. It indicates that, the connectivity coefficients are directly related to the change in the steady state response of the output signal caused by a unit variation in the input, and changing the connectivity coefficients is equivalent to shifting the response up or down; however, the time constants mainly present a time-varying behavior, a faster system has low time constant, while a slower system has higher time constant.

Furthermore, in order to hold a material balance of the injected fluid, the following inequality constraint should be satisfied:

$$
\sum_{i}^{N_{\text {inj }}} f_{i j} \leq 1, \quad j=1,2, \cdots, N_{\text {pro }}
$$

\section{(2) Injector-producer pair-based representation -CRMIP}

As shown in Fig. 3, the CRMIP representation assigns one time constant and one connectivity coefficient for each injector (i)-producer $(j)$ pair, which is more suitable for estimation of interwell connectivity in heterogeneous waterflood reservoir. The governing differential equation for each injector-producer pair based control volume is states as:

$\frac{d q_{i j}(t)}{d t}+\frac{1}{\tau_{i j}} q_{i j}(t)=\frac{1}{\tau_{i j}} f_{i j} I_{i}(t)-J_{i j} \frac{d P_{w f, j}}{d t}$

where $q_{i j}(t)$ is the production rate of producer $j$ relative to the $i-j$ pair control volume at time $t, \mathrm{~m}^{3} / \mathrm{d} ; \tau_{i j}$ is the time constant of the $i-j$ pair control volume; $I_{i}(t)$ is the injection rate of injector $i$ at time $t$, $\mathrm{m}^{3} / \mathrm{d} ; J_{i j}$ is the productivity index relative to the $i-j$ pair control volume, $\mathrm{m}^{3} /(\mathrm{MPa} \cdot \mathrm{d}) ; P_{w f, j}$ is the bottom-hole pressure of producer $j$ at time $t, \mathrm{MPa} ; f_{i j}$ is the connectivity coefficient associated to the $i-j$ pair control volume, $\in[0,1]$.

Based on superposition principle in time and space, the semianalytical solution Eq. (4) at time interval $t_{k}$ is determined, which takes the form of Eq. (5) (Sayarpour et al., 2007).

$$
\begin{aligned}
q_{i j}\left(t_{k}\right)= & q_{i j}\left(t_{0}\right) e^{-\left(\frac{t_{k}-t_{0}}{\tau_{i j}}\right)} \\
& +\sum_{s=1}^{k}\left[\left(1-e^{-\frac{\Delta t_{s}}{\tau_{i j}}}\right)\left(f_{i j} \cdot I_{i}^{(s)}-J_{i j} \cdot \tau_{i j} \frac{\Delta p_{w f, j}^{(s)}}{\Delta t_{s}}\right) e^{-\left(\frac{t_{k}-t_{s}}{\tau_{i j}}\right)}\right]
\end{aligned}
$$

At time interval $t_{\mathrm{k}}$, the total production rate $q_{j}\left(t_{k}\right)$ of producer $j$ are described as:

$q_{j}\left(t_{k}\right)=\sum_{i=1}^{N_{i n j}} q_{i j}\left(t_{k}\right)$

When neglecting aquifer support besides injectors and changes of bottom-hole pressure, for each injector-producer pair, three unknown model parameters exist: $f_{i j}, q_{i j}\left(t_{0}\right)$ and $\tau_{i j}$. The model unknowns of the CRMIP representation sums to $3 \times N_{\text {pro }} \times N_{i n j}$. Apart from the inequality constraint shown in Eq. (3), the initial values of liquid production rate $q_{i j}\left(t_{0}\right)$ of each injector-producer pair based control volume must hold the following equality constraint.

$$
\sum_{i}^{N_{i n j}} q_{i j}\left(t_{0}\right)=q_{j}\left(t_{0}\right)
$$

\subsection{Koval fractional-flow equation}

Due to the fact that the CRM representations are merely singlephase flow model, which history matches and predicts the total production rates, hence a fractional flow model must be implemented into the CRM representations to separate oil production 


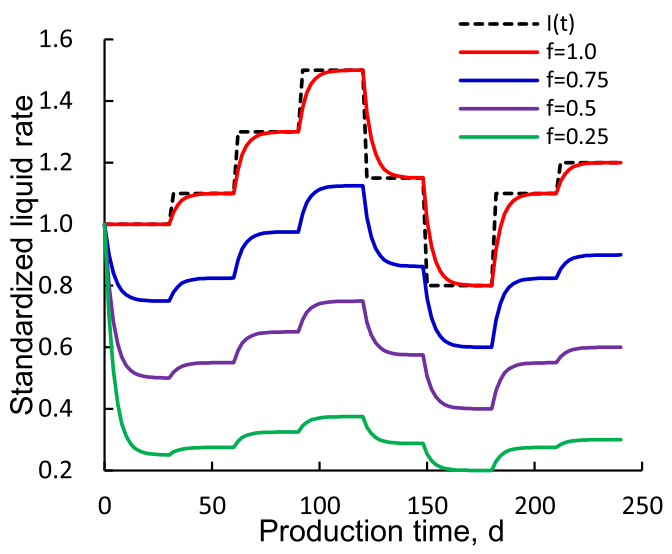

(a) Impact of connectivity coefficient

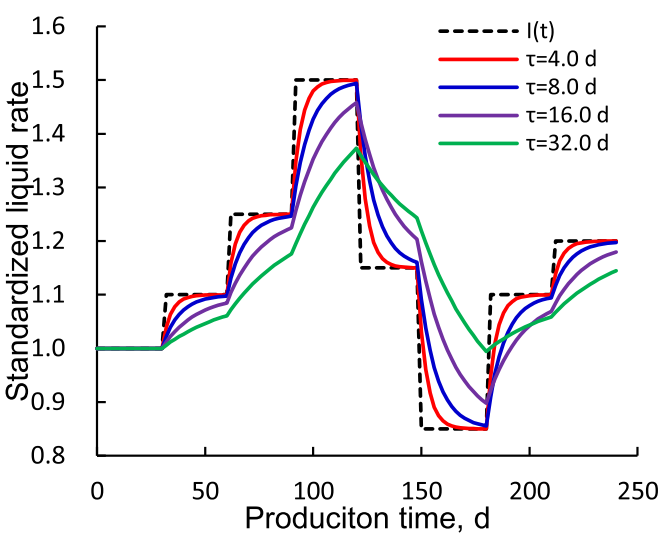

(b) Impact of time constant

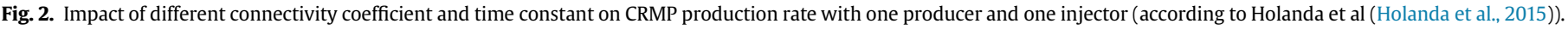

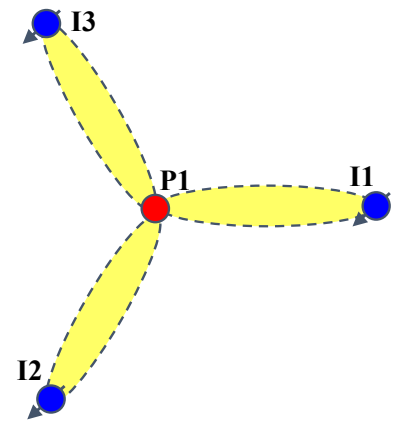

Fig. 3. Schematic of reservoir control volume in the CRMIP representation.

from the total production. For this study, to address the issue, the fractional-flow equation inferred from field production data using the Koval theory is advanced to develop a relationship between water cut and dimensionless time. By history matching field water cut data, two characteristic parameters, the Koval factor and drainage volume, are estimated, which takes the form of Eq. (8) (Cao et al., 2014, 2015).

$f_{w}=\left\{\begin{array}{cc}0 & t_{D}<\frac{1}{K_{v a l}} \\ \frac{K_{v a l}-\sqrt{\frac{K_{v a l}}{t_{D}}}}{K_{v a l}-1} & \frac{1}{K_{v a l}}<t_{D}<K_{v a l} \\ 1 & t_{D} \geq K_{v a l}\end{array}\right.$

where $K_{v a l}$ denotes the Koval factor, which is a measure of heterogeneity, the stronger the local heterogeneity, the larger the Koval factor is. For homogeneous reservoirs, $K_{v a l}$ is equal to $1.0 ; t_{D}$ denotes the dimensionless time, which is defined to be the cumulative water injection in pore volumes, given by

$t_{D}=\frac{\sum_{k} \sum_{i} f_{i j} I_{i}}{V_{p j}}$

where $f_{i j}$ is the connectivity coefficient, easily obtained by history matching field production data using the above-mentioned CRM representations; $V_{p j}$ is the drained pore volume of a producer or each injector-producer pair, $\mathrm{m}^{3} ; I_{i}$ is the injection contribution to the reservoir control volume at time $t_{k}, \mathrm{~m}^{3} / \mathrm{d}$. For further details, the reader is referred to Cao et al. $(2014,2015)$.

\section{Ensemble-based optimization process}

To obtain a satisfactory estimation of interwell connectivity in waterflooding reservoirs, an efficient ensemble-based technique is addressed to history matching the observed production data, such as liquid production rate, oil production rate and water cut, and the estimated results are significantly influenced by characteristic parameters coupling with different reservoir control volumes. On basis of the theory that predicted values of production performance should be in accordance with the observed, a least-squared objective function needs to be developed, and take the form of Eq. (10).

$\underset{\boldsymbol{u} \in R^{N_{u}}}{\operatorname{minimize}} J(\mathbf{u})=\sum_{k=1}^{N_{t}} \sum_{j=1}^{N_{\text {pro }}}\left[q_{j}^{\mathrm{cal}}\left(t_{k}\right)-q_{j}^{\mathrm{obs}}\left(t_{k}\right)\right]^{2}$

Subjected to,

$u_{i}^{\text {low }} \leq u_{i} \leq u_{i}^{u p}, \quad i=1,2, \ldots, N_{u}$

$c_{i}(\mathbf{u}) \leq 0, \quad i=1,2, \ldots, n_{i}$

$e_{i}(\mathbf{u})=0, \quad i=1,2, \ldots, n_{e}$

where $J(\mathbf{u})$ is the least-squared objective function; $\mathbf{u}$ is the $1 \times N_{u}$ vector of unknown controlling variables; Eqs. (10b), (10c) and (10d) are the bound, inequality, and equality constraints, respectively; $u_{i}^{\text {low }}$ and $u_{i}^{u p}$ are the lower and upper limits for the $i$ th controlling variable $u_{i}$, respectively; $n_{i}$ and $n_{e}$ are the number of inequality and equality constraints, respectively.

In order to enforce the upper and lower bound constraints, we apply a logarithm transformation (Gao and Reynolds, 2006) to each element of the unknown controlling vector, and the $i$ th component of the transformed controlling vector $\mathbf{v}$ corresponding to $\mathbf{u}$ is given by

$v_{i}=\ln \left(\frac{u_{i}-u_{i}^{\text {low }}}{u_{i}^{u p}-u_{i}}\right)$

where $u_{i}$ denotes the $i$ th component of the unknown controlling vector $\mathbf{u}$. After applying this log-transformation to each of the 
controlling variables, the original bound-constrained optimization problem is transformed to an unbounded optimization problem because each $v_{i}$ can vary from $-\infty$ to $+\infty$. However, following Oliveira and Reynolds (2014) in the log-domain, the variables are still truncated to the interval $[-5,5]$ as the practical limits in the log domain.

After the optimization problem is solved for $\mathbf{v}$, the unknown controlling vector $\mathbf{u}$ can be obtained with the inverse logtransformation, which can be expressed as

$u_{i}=\frac{\exp \left(v_{i}\right) u_{i}^{u p}+u_{i}^{\text {low }}}{1+\exp \left(v_{i}\right)}=\frac{u_{i}^{u p}+\exp \left(-v_{i}\right) u_{i}^{\text {low }}}{1+\exp \left(-v_{i}\right)}$

In general, the inequality and equality constraints (Eqs. (10c) and (10d), respectively) are handled with the augmented Lagrangian method, given by

$\boldsymbol{A}_{T} \boldsymbol{u}-\boldsymbol{C} \leq 0$

$\boldsymbol{A}_{\xi} \boldsymbol{u}-\boldsymbol{E}=0$

where $A_{T}$ is the $n_{i} \times N_{u}$ Jacobian matrix of inequality constraint functions; $A_{\xi}$ is the $n_{e} \times N_{u}$ Jacobian matrix of equality constraint functions; $\boldsymbol{C}=\left(C_{1}, C_{2}, \ldots C_{n_{i}}\right)^{T}$ is the $n_{i}$-dimensional column vector containing the inequality constraint values; $\boldsymbol{E}=\left(E_{1}, E_{2}, \ldots E_{n_{e}}\right)^{T}$ is the $n_{e}$-dimensional column vector containing the equality constraint values.

After using the log-transformation to handle bound constraints, the augmented Lagrangian function (Oliveira and Reynolds, 2014) is defined as

$$
\begin{aligned}
L_{a}(\mathbf{u}, \lambda, \mu)= & J(\mathbf{u})-\sum_{j=1}^{n_{e}} \lambda_{e, j}\left[s_{e, j} \cdot e_{j}(\mathbf{u})\right]+\frac{1}{2 \mu} \sum_{j=1}^{n_{e}}\left[s_{e, j} \cdot e_{j}(\mathbf{u})\right]^{2} \\
& -\sum_{i=1}^{n_{i}} \lambda_{c, i} \max \left[s_{c, i} \cdot c_{i}(\mathbf{u}),-\mu \cdot \lambda_{c, i}\right] \\
& +\frac{1}{2 \mu} \sum_{i=1}^{n_{i}}\left\{\max \left[s_{c, i} \cdot c_{i}(\mathbf{u}),-\mu \cdot \lambda_{c, i}\right]\right\}^{2}
\end{aligned}
$$

where $\lambda_{e, j}$ and $\lambda_{c, i}$ are the Lagrange multipliers associated with equality and inequality constraints, respectively; $\mu$ is the penalty parameter; $s_{e j}$ and $s_{c, i}$ are the scaling factors for equality and inequality constraints, respectively, which will be applied to scale the constraints and to avoid convergence issues caused by poor scaling; when the $C_{i}$ and $E_{j}$ are the nonzero constraint values in Eq. (14), we define $s_{e, j}=1 / E_{j}$ for $j=1,2, \ldots, n_{e}$ and $s_{c, i}=1 / C_{i}$ for $i=1,2, \ldots, n_{i}$. Note that, if we apply the log-transformation to alleviate the bound constraint, and the general inequality and equality constraints do not exist, the augmented Lagrangian function reduces to a traditional unbounded optimization procedure.

Through the so-called outer loop, the Lagrange multiplier $\lambda$ and the penalty parameter $\mu$ are self-adaptively adjusted during optimization. Once they are fixed, we solve the following sub-problem.

$\underset{\boldsymbol{u} \in R^{N_{u}}}{\operatorname{minimize}} L_{a}(\mathbf{u})$

Lagrange multiplier $\lambda$ or the penalty parameter $\mu$ in the next outer iteration according to the magnitude of any constraint violation. If the constraint violation is large, we keep the same Lagrange multiplier $\lambda$ fixed and decrease the penalty parameter $\mu$. Otherwise, when the constraint violation is small, we update the Lagrange multiplier $\lambda$ and keep the same penalty parameter $\mu$.

For the augmented Lagrangian method, the constraint violations are measured using the constraint violation factor $\sigma_{c_{v}}$, which is defined as

$$
\begin{gathered}
\sigma_{c_{v}}=\left\{\sqrt{\frac{1}{n_{v}}\left\{\sum_{j=1}^{n_{e}}\left[s_{e, j} \cdot e_{j}(\mathbf{u})\right]^{2}+\sum_{i=1}^{n_{i}}\left\{\max \left[s_{c, i} \cdot c_{i}(\mathbf{u}), 0\right]\right\}^{2}\right\},}\right. \\
n_{v}>00, n_{v}=0
\end{gathered}
$$

where $n_{v}$ denotes the number of violated constraints; Given $\eta^{l}$, the constraint-violation tolerance at outer iteration $l$, the Lagrange multipliers are updated whether $\sigma_{c_{v}}<\eta^{l}$ is satisfied.

$\lambda_{e, j}^{l+1}=\lambda_{e, j}^{l}+\frac{s_{e, j} \cdot e_{j}\left(\mathbf{u}^{l}\right)}{\mu^{l}}, \quad j=1,2, \ldots, n_{e}$

$\lambda_{c, i}^{l+1}=\max \left[0, \lambda_{c, i}^{l}+\frac{s_{c, i} \cdot c_{i}\left(\mathbf{u}^{l}\right)}{\mu^{l}}\right], \quad i=1,2, \ldots, n_{i}$

If $\sigma_{c_{v}}>\eta^{l}$, then the penalty parameter $\mu$ is updated by

$\mu^{l+1}=\tau \cdot \mu^{l}$

For this study, $\tau=0.25$ and $\eta^{l}=0.01$.

The initial guess of $\mu^{0}$ is obtained by requiring that, when all squared constraint terms in Eq. (14) are equal to 0.1, the sum of these terms times $1 /(2 \mu)$ is of the same magnitude as the augmented objective function, which implies that

$\mu^{0}=\frac{0.1 \times\left(n_{e}+n_{i}\right)}{2 J\left(\mathbf{u}^{0}\right)}$

Following Chen et al. (2012), the initial guess of Lagrange multipliers are as follows,

$\lambda_{e, j}^{0}=\frac{s_{e, j} \cdot e_{j}\left(\mathbf{u}^{0}\right)}{\mu^{0}}, \quad j=1,2, \ldots, n_{e}$

$\lambda_{c, i}^{0}=\max \left[0, \frac{s_{c, i} \cdot c_{i}\left(\mathbf{u}^{0}\right)}{\mu^{0}}\right], \quad i=1,2, \cdots, n_{i}$

At each iteration of the so-called inner loop, the newly developed StoSAG optimization algorithm (Fonseca et al., 2016) is applied to solve the above-mentioned augmented Lagrangian function by considering hybrid nonlinear constraints.

$\mathbf{u}^{k+1}=\mathbf{u}^{k}-a_{k}\left[\frac{\mathbf{d}_{k}}{\left\|\mathbf{d}_{k}\right\|_{\infty}}\right]$

for $\mathrm{k}=0,1,2, \ldots$ until convergence, where $\mathbf{u}^{0}$ is the initial guess and $\mathbf{u}^{k}$ is the estimate of the optimal controlling vector at the $k$ th iteration; $a_{k}$ is the step size; and the initial value $a_{k}^{0}=0.1 \times \min _{i}\left(u_{i}^{u p}-u_{i}^{\text {low }}\right) ; \mathbf{d}_{k}$ denotes the search direction vector, which is used to provide a reasonable approximation to the singlysmoothed true gradient of the augmented Lagrangian function $\boldsymbol{L}_{a}\left(\mathbf{u}^{k}\right) ;\|\cdot\|_{\infty}$ denotes the infinite norm of the controlling vector.

In this work, provided that the unknown control variables including connectivity coefficients, time constants and productivity indices are usually assumed to be independent and treated as constant, no correlation of different unknown control variables should be considered. To obtain the search direction $\mathbf{d}_{k}$ at iteration $k+1$, we first generate $N_{e}$ samples of the Gaussian random vector $\mathbf{u}^{k}$, where $\boldsymbol{X} \sim N\left(\mathbf{u}^{k}, \boldsymbol{I}\right)$ (i.e., the mean of $\mathbf{u}^{k}$ is equal to $\mathbf{u}^{k}$ and its covariance matrix $\boldsymbol{I})$. These samples can be generated as, 
$\widetilde{\mathbf{u}_{j}^{k}}=\mathbf{u}^{k}+Z_{j}, \quad j=1,2, \cdots N_{e}$

where $k$ is the iteration number of inner loop; $\boldsymbol{Z}_{j}$ is an $N_{u}$-dimensional independent, standard, random-normal vector with mean equal to $N_{u}$-dimensional zero vector and covariance matrix equal to $N_{u} \times N_{u}$ identity matrix $I$, i.e., $\boldsymbol{Z}_{j} \sim N(0, \quad \boldsymbol{I})$.

$$
\text { Specifically, } \quad \text { assuming } \quad \delta \mathbf{U}_{j}^{k}=\widetilde{\mathbf{u}_{j}^{k}}-\mathbf{u}^{k} \quad \text { and }
$$
$\left.\delta \boldsymbol{L}_{a}\right|_{j} ^{k}=\boldsymbol{L}_{a}\left(\mathbf{u}_{j}^{k}\right)-\boldsymbol{L}_{a}\left(\mathbf{u}^{k}\right)$, the search direction vector $\mathbf{d}_{k, s t o}$ of StoSAG optimization can be written as

$\mathbf{d}_{k, s t o}=\left.\frac{1}{N_{e}} \sum_{j=1}^{N_{e}}\left(\widetilde{\mathbf{U}_{j}^{k}}\left(\widetilde{\delta \mathbf{U}_{j}^{k}}\right)^{T}\right)^{+} \widetilde{\mathbf{U}_{j}^{k}} \cdot \delta \mathbf{L}_{a}\right|_{j} ^{k}$

where the superscript ' + ' on a matrix denotes the Moore-Penrose pseudo-inverse; the superscript ' $T$ ' on a vector or matrix denotes the transpose process.

For StoSAG optimization, if $\boldsymbol{L}_{a}\left(\mathbf{u}^{k+1}\right)<\boldsymbol{L}_{a}\left(\mathbf{u}^{k}\right)$, we accept $\mathbf{u}^{k+1}$ as the new iterate; otherwise, we decrease the step size by replacing $a_{k}$ with $0.5 a_{k}$ and apply Eq. (21) again until we obtain a reasonable search direction vector $\mathbf{u}^{k+1}$ such that $\boldsymbol{L}_{a}\left(\mathbf{u}^{k+1}\right)<\boldsymbol{L}_{a}\left(\mathbf{u}^{k}\right)$ or we reach the maximal number of allowable step-size cuts, which is equal to 10 in this work. If, with the maximal number of step-size cuts allowed, a reasonable $\mathbf{u}^{k+1}$ has not been found satisfying $\boldsymbol{L}_{a}\left(\mathbf{u}^{k+1}\right)<\boldsymbol{L}_{a}\left(\mathbf{u}^{k}\right)$, then we generate a new sample of perturbed controls from Eq. (22) to generate a new approximation of the singly-smoothed true gradient to find an uphill search direction. If, with five successive samples of perturbed controls, an uphill direction is not found, then the inner loop is terminated. In addition, we assume the maximal number of allowable simulation runs as 2000 in the synthetic case presented. At iteration $l$ of the outer loop, the convergence criteria for the inner loop are described as.
$\frac{\left|L_{a}\left(\mathbf{u}^{k+1}\right)-L_{a}\left(\mathbf{u}^{k}\right)\right|}{\max \left[\left|L_{a}\left(\mathbf{u}^{k}\right)\right|, 1.0\right]} \leq \xi_{f}^{l}$

$\frac{\left\|\mathbf{u}^{k+1}-\mathbf{u}^{k}\right\|_{2}}{\max \left(\left\|\mathbf{u}^{k}\right\|_{2}, 1.0\right)} \leq \xi_{u}^{l}$

Note that, both $\xi_{f}^{l}$ and $\xi_{u}^{l}$ vary with outer iterations, in which we always use in this work that $\xi_{f}^{0}=0.1$ and $\xi_{u}^{0}=0.1$. For every outer iteration, the adjustment strategies of $\xi_{f}^{l}$ and $\xi_{u}^{l}$ are given by

$\xi_{f}^{l}=\max \left(0.5 \xi_{f}^{l-1}, \xi_{f}^{h}\right)$

$\xi_{u}^{l}=\max \left(0.5 \xi_{u}^{l-1}, \xi_{u}^{h}\right)$

For the steepest descent, if at iteration $l$ of the outer loop, the inner iteration converges to $\mathbf{u}^{*}$, then we set $\mathbf{u}_{\mathrm{opt}}^{l+1}=\mathbf{u}^{*}$. The complete augmented Lagrangian algorithm will be terminated if both of the following equations holds.

$\frac{\left|L_{a}\left(\mathbf{u}_{\mathrm{opt}}^{l+1}\right)-L_{a}\left(\mathbf{u}_{\mathrm{opt}}^{l}\right)\right|}{\max \left[\left|L_{a}\left(\mathbf{u}_{\mathrm{opt}}^{l}\right)\right|, 1.0\right]} \leq \xi_{f}^{h}$

$\frac{\left\|\mathbf{u}_{\mathrm{opt}}^{l+1}-\mathbf{u}_{\mathrm{opt}}^{l}\right\|_{2}}{\max \left(\left\|\mathbf{u}_{\mathrm{opt}}^{l}\right\|_{2}, 1.0\right)} \leq \xi_{u}^{h}$

where $\xi_{f}^{h}$ and $\xi_{u}^{h}$ are the presumed final tolerances for the augmented Lagrangian algorithm, respectively. In the cases, $\xi_{f}^{h}=10^{-4}$ and $\xi_{u}^{h}=10^{-3}$, following Oliver et al. (2008).

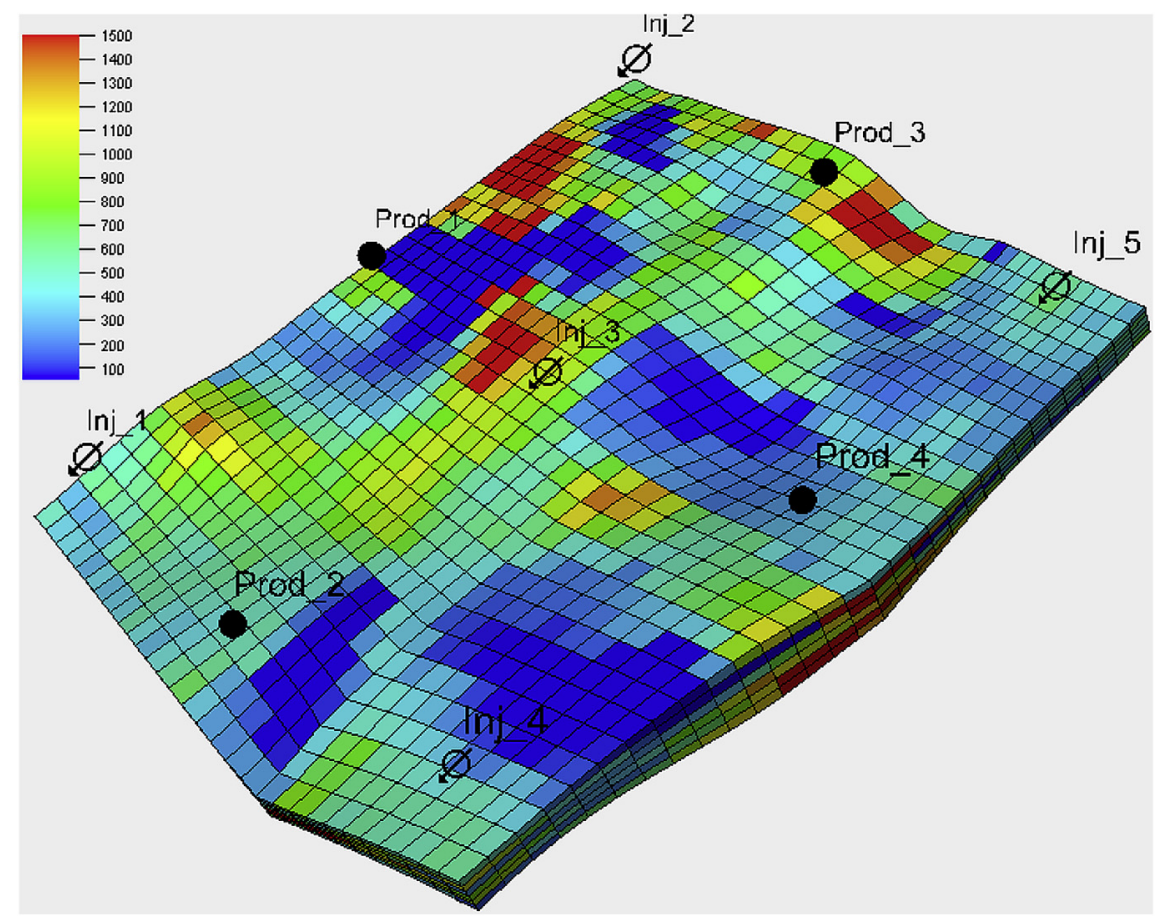

Fig. 4. The 3D geological model of the synthetic heterogeneous reservoir. 
Table 1

Reservoir and fluid parameters of the three-dimensional reservoir simulation model.

\begin{tabular}{lll}
\hline Parameter & Value & Parameter \\
\hline Initial reservoir pressure, MPa & 5.5 & Water viscosity, mPa $\cdot \mathrm{s}$ \\
Reservoir temperature, C & 36 & Oil viscosity, mPa $\cdot \mathrm{s}$ \\
Average porosity & 0.28 & Water density, $\mathrm{kg} / \mathrm{m}^{3}$ \\
Average permeability, $10^{-3} \mu \mathrm{m}^{2}$ & 676 & Oil density, $\mathrm{kg} / \mathrm{m}^{3}$ \\
Irreducible water saturation $(\mathrm{Swc})$ & 0.265 & Rock compressibility, $1 / \mathrm{MPa}$ \\
Oil relative permeability at Swc & 1.0 & Oil compressibility, $1 / \mathrm{MPa}$ \\
Residual oil saturation (Sor) & 0.32 & Water compressibility, $1 / \mathrm{MPa}$ \\
Water relative permeability at Sor & 0.4 & Bottom hole pressure, MPa \\
\hline
\end{tabular}

\section{Case study}

To validate the reliability of the proposed optimization framework for estimation of interwell connectivity, a three-dimensional (3D) synthetic reservoir simulation model is established, as shown in Fig. 4. The parameters of the 3D geological model are as follows: a corner-point grid system is adopted, with a $31 \times 31 \times 6$ grid, and the step size of $X, Y$, and $Z$ direction are $29 \mathrm{~m}, 30 \mathrm{~m}$ and $1.2 \mathrm{~m}$, respectively. The reservoir and fluid parameters are listed in Table 1. Five-spot injection production pattern is utilized to exploit the heterogeneous synthetic reservoir, and there are 9 vertical wells (5

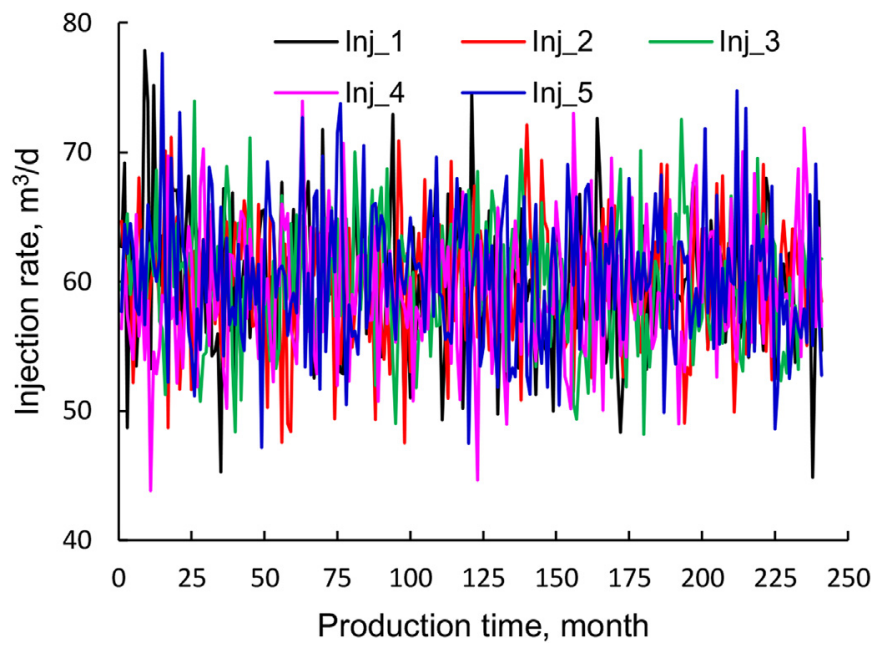

Fig. 5. The inputted values of monthly-averaged injection rate.

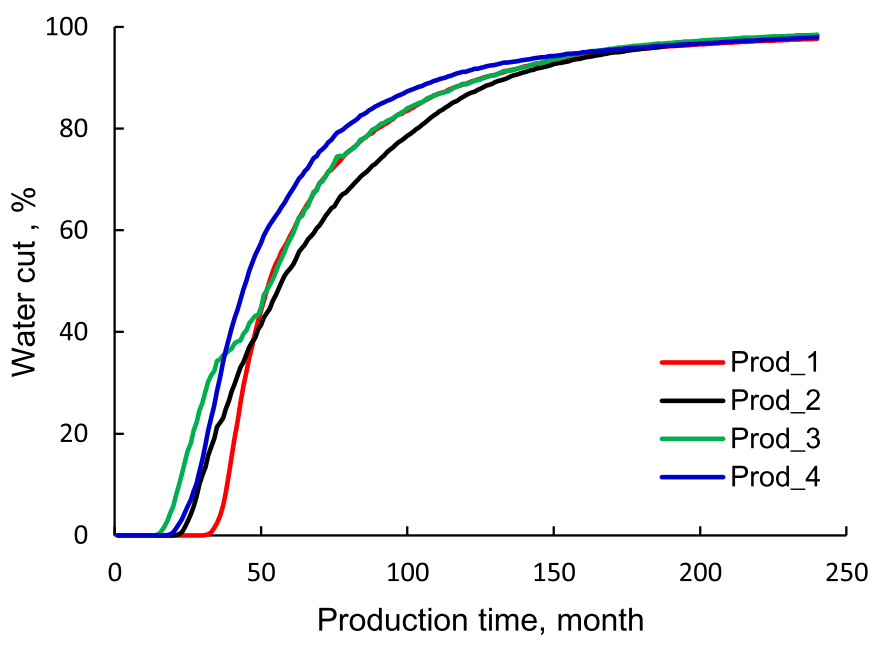

Fig. 6. The simulated water cut data of each producer. injectors and 4 producers) totally. Production is performed at a constant bottom-hole pressure of $3.0 \mathrm{MPa}$, and injection is performed at a varying surface liquid rate, as presented in Fig. 5, which is random-normally distributed with mean equal to $60 \mathrm{~m}^{3} / \mathrm{d}$ and variance equal to 5 . Meanwhile, the fluid support besides injectors, such as an aquifer influx, along with the impact of gravity and capillary pressure are neglected. The commercial reservoir simulator Eclipse is introduced to achieve the observed production data for 240 months, and the ultimate water cut reaches to $98.1 \%$. The simulated water cut data of each producer and production performance of the synthetic reservoir are shown in Figs. 6 and 7, respectively.

The coefficient of determination (or $R^{2}$ ) is developed to indicate the goodness of fitting between the observed and predicted

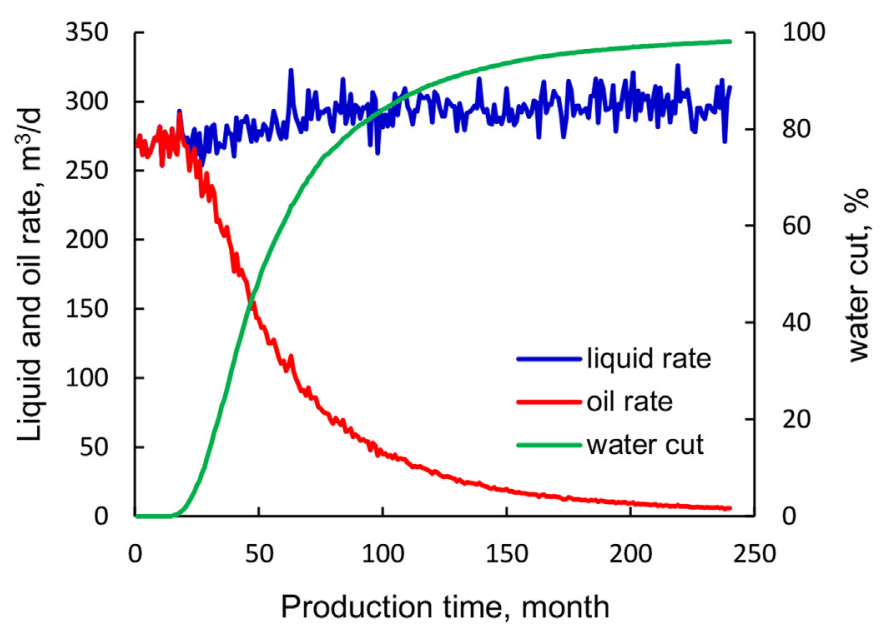

Fig. 7. The actual production performance of the entire reservoir.

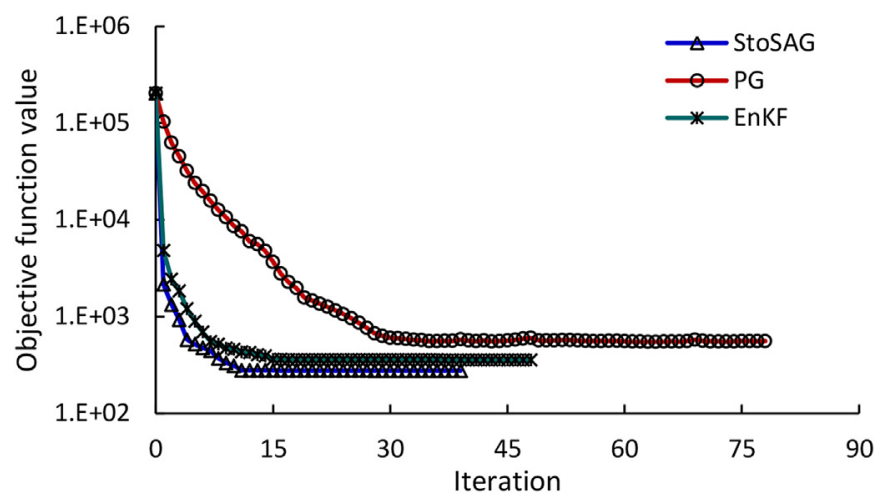

Fig. 8. The iteration convergence process of objective function under different optimization algorithms. 
production data. An $R^{2}$ of 1.0 indicates that the predicted production data perfectly fits the observed values, which is defined as

$R^{2}=1-\frac{\sum_{i}\left(y_{i}-f_{i}\right)^{2}}{\sum_{i}\left(y_{i}-\bar{y}\right)^{2}}$

where $y_{i}$ is the observed value; $f_{i}$ is the predicted value; $\bar{y}$ is the mean of the observed data.

\subsection{Robustness of optimization framework}

To understand the robustness of the developed optimization framework definitely for hybrid nonlinear constrained problems, CRMP representation associated with each producer-based control volume is utilized to calculate liquid production rate, afterwards, the StoSAG, EnKF and traditional projected gradient (PG) methods are performed to history match the field production data, respectively. Comparisons of the iteration convergence process and fitting effect of the observed liquid production data are further carried out, as shown in Figs. 8-9.

From Figs. 8-9, we observe that, both StoSAG, EnKF and traditional PG methods can achieve a satisfactory fitting of the observed production data, and convergence speed of StoSAG optimization tends to be faster. The main reason is that, the projected-gradient method has to be done in an iterative form and require extensive computational resources, however, the StoSAG method can be used to compute gradients through a Monte Carlo evaluation of the approximate gradient over an ensemble of state variables corresponding to their uncertainties by a simpler means than that of the EnKF method, which enables the StoSAG optimization algorithm to handle the sequential data assimilation in large-scale nonlinear dynamics more robustly.

\subsection{Comparison of different CRM representations}

Depending on the heterogeneity of reservoirs being studied, different injectors can influence the production rate of a certain producer with various waterflood front velocities. Therefore, assuming only one time constant for each producer, it does not agree well with the actual reservoir condition completely, which leads to the CRMP no longer a reliable model and some systematic errors will be incorporated in description of the dynamic behaviors to drive the evolution of connectivity inevitably. In such a case, it may be better to apply the injector-producer based representation (CRMIP) to characterize the real-time liquid production rate. For this study, sensitivity analysis of different CRM representations, CRMP and CRMIP, are implemented to compare the estimated results of interwell connectivity in the synthetic waterflood reservoir. Fitting effect of the observed liquid production data using the CRMP and CRMIP models are displayed in Figs. 10 and 11, respectively. The corresponding estimates of interwell connectivity are shown in Fig. 12.

It demonstrates that, compared to CRMP, the CRMIP holds more degrees of freedom to capture the reservoir's dynamic behaviors; Even CRMIP does not match the production history greater than CRMP, it still follows most trends of the system dynamics while CRMP tends to provide a smoother production rate estimates due to

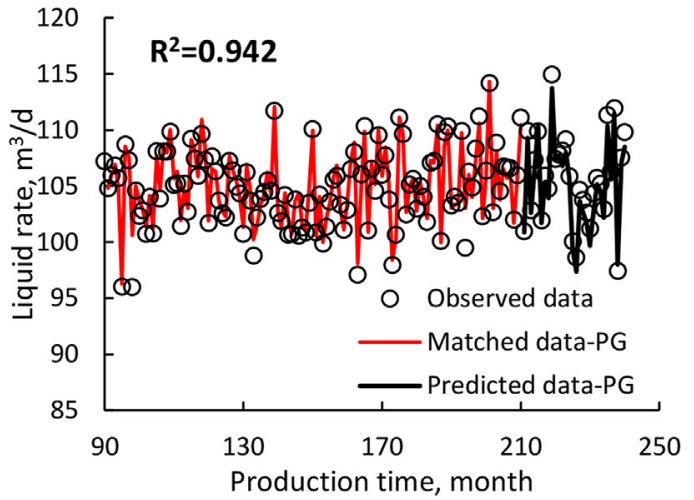

(a) PG optimization

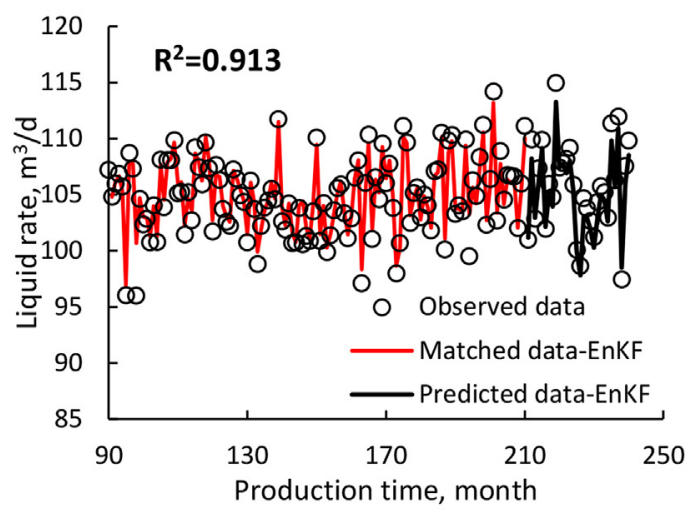

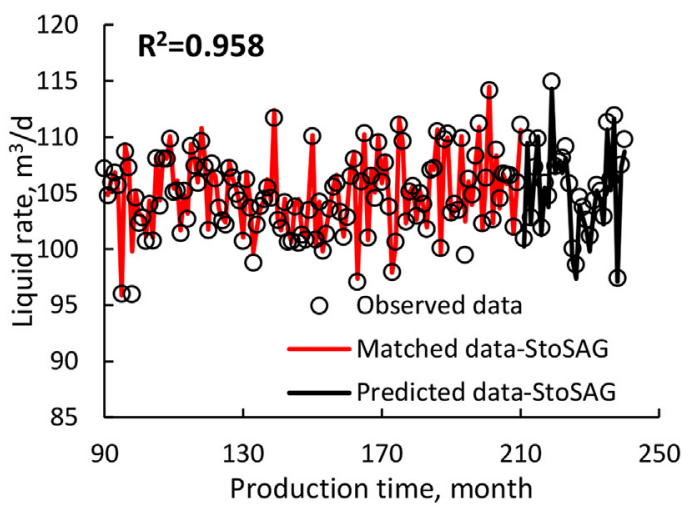

(b) StoSAG optimization

(c) EnKF Optimization

Fig. 9. Liquid rate fitting effect of typical well Prod_4 using different optimization algorithms. 


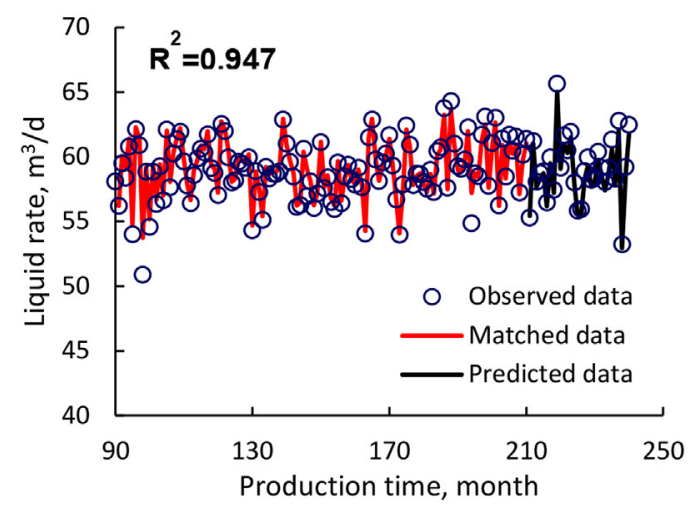

(a) Prod_1

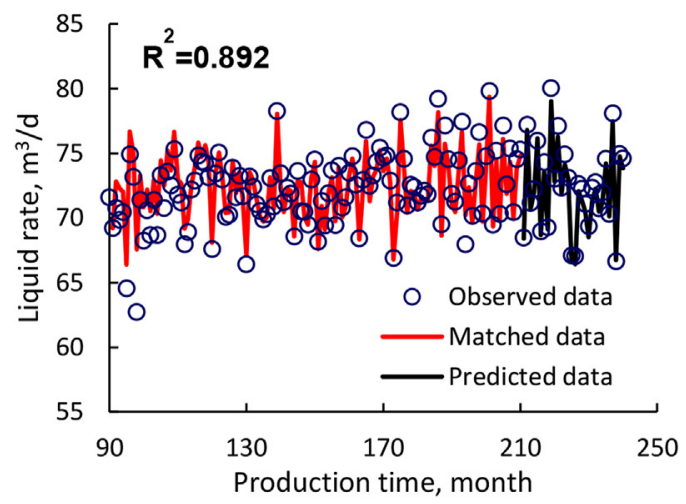

(c) Prod_3

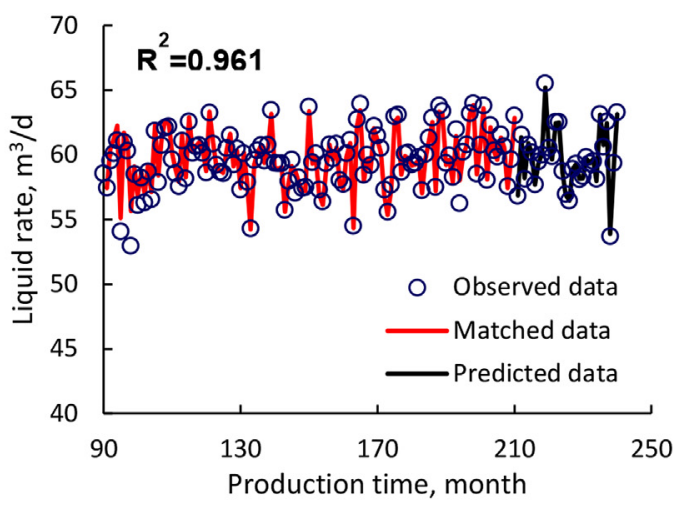

(b) Prod 2

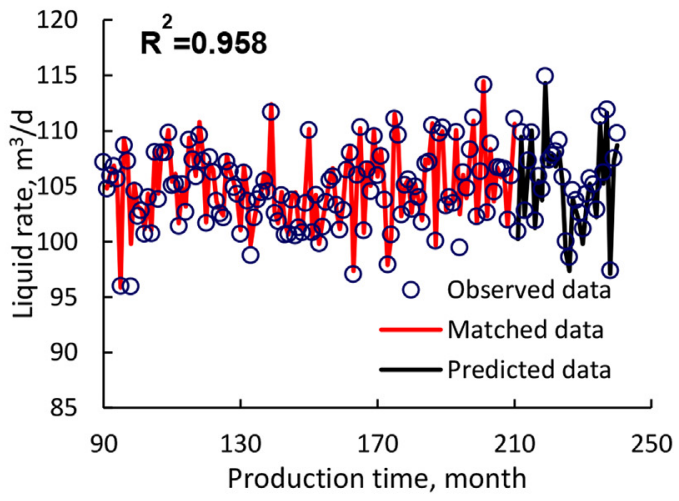

(d) Prod 4

Fig. 10. Fitting effect of liquid production data using the CRMP representation.

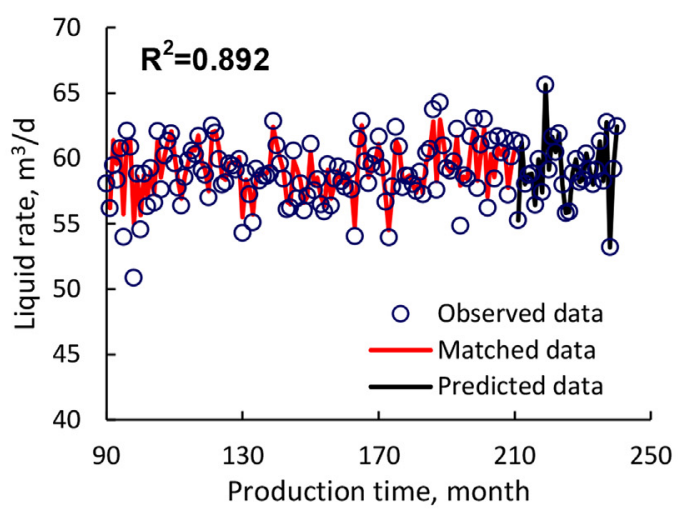

(a) Prod 1

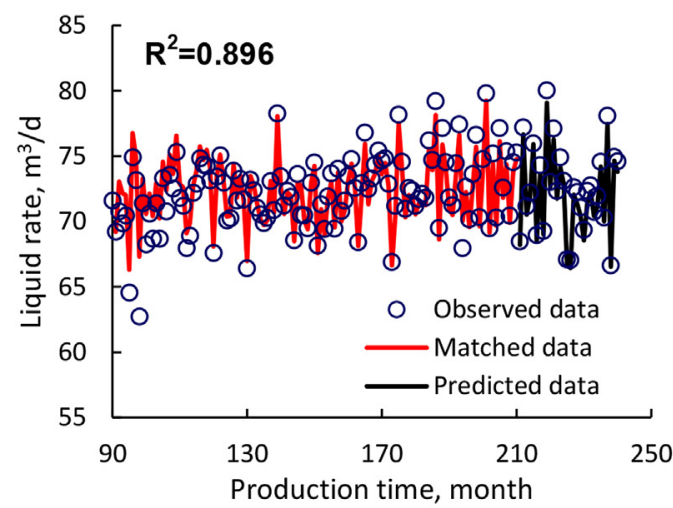

(c) Prod_3

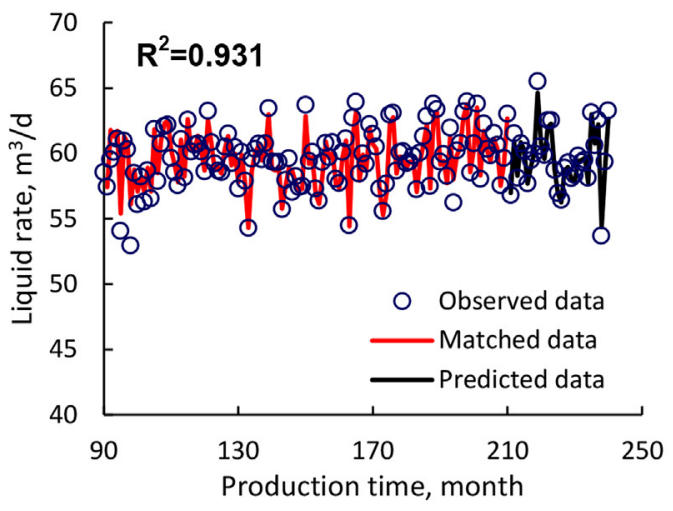

(b) Prod 2

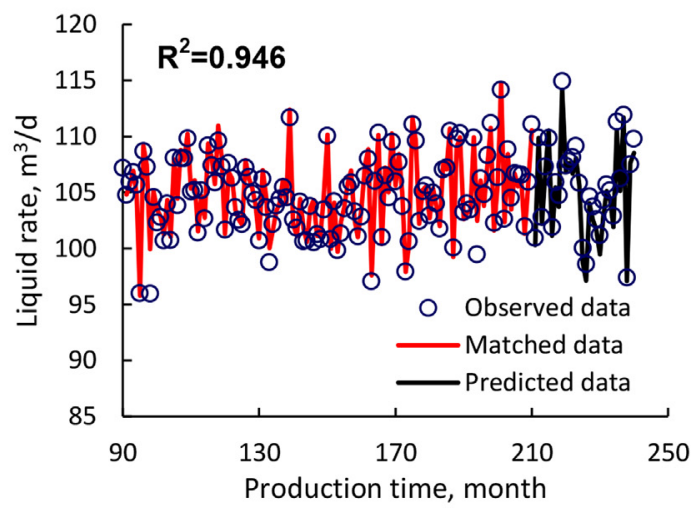

(d) Prod 4

Fig. 11. Fitting effect of liquid production data using the CRMIP representation. 


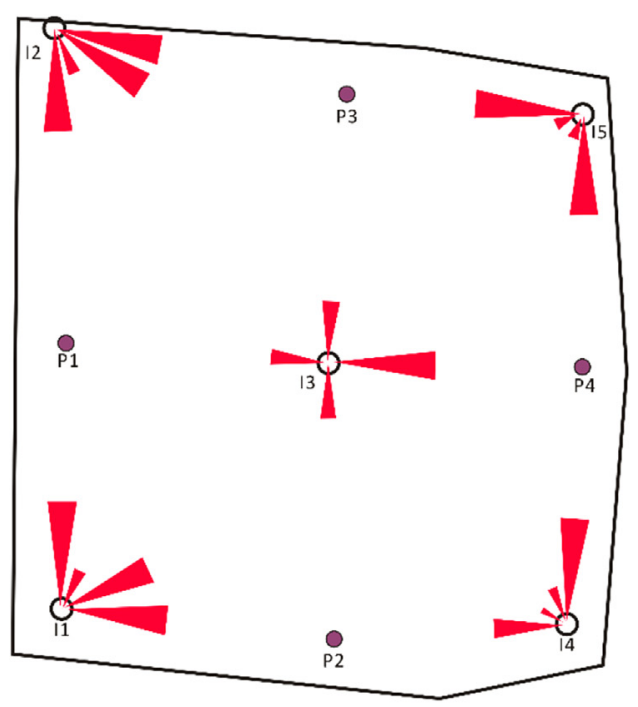

(a) CRMP model

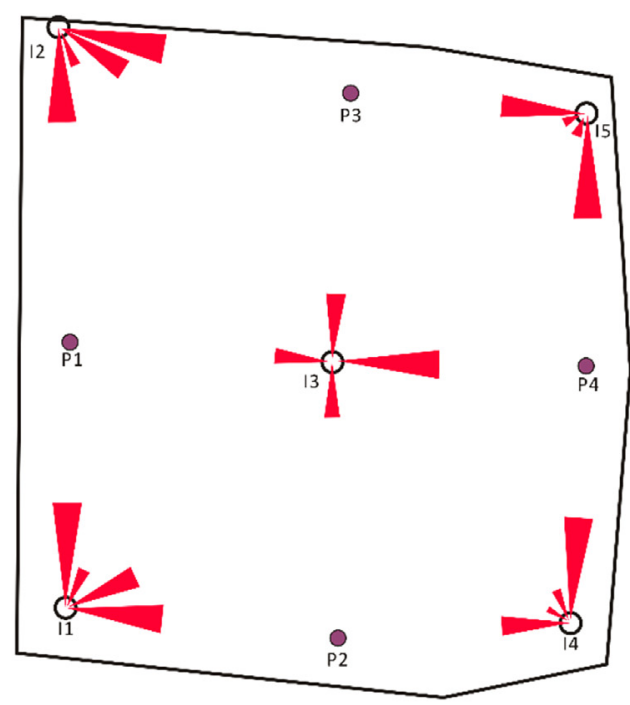

(b) CRMIP model

Fig. 12. The estimated interwell connectivities of synthetic reservoir using different CRM representations.

Table 2

Other estimated geological parameters of the synthetic waterflooding reservoir.

\begin{tabular}{|c|c|c|c|c|c|c|c|c|}
\hline & \multicolumn{4}{|c|}{ Time constant, $\mathrm{d}$} & \multicolumn{4}{|c|}{ Drained pore volume, $\mathrm{m}^{3}$} \\
\hline & Prod_1 & Prod_2 & Prod_3 & Prod_4 & Prod_1 & Prod_2 & Prod_3 & Prod_4 \\
\hline Inj_1 & 19.2 & 14.9 & 8.5 & 7.0 & 599.0 & 1448.7 & 381.9 & 1270.5 \\
\hline Inj_2 & 19.1 & 13.3 & 7.9 & 7.1 & 1000.5 & 558.2 & 1052.0 & 1350.7 \\
\hline Inj_3 & 18.7 & 11.8 & 7.5 & 7.2 & 927.9 & 691.6 & 868.4 & 1457.2 \\
\hline Inj_4 & 17.8 & 10.4 & 7.2 & 7.6 & 574.6 & 910.0 & 674.3 & 1082.1 \\
\hline Inj_5 & 16.4 & 9.3 & 7.1 & 8.0 & 606.6 & 320.2 & 2139.1 & 651.9 \\
\hline
\end{tabular}

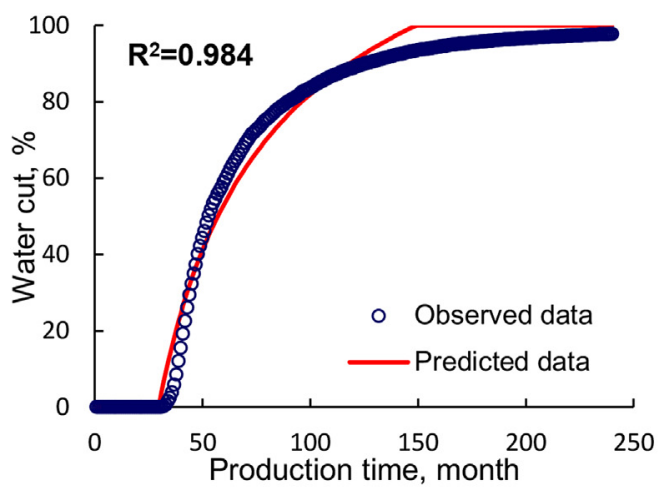

(a) Prod_1

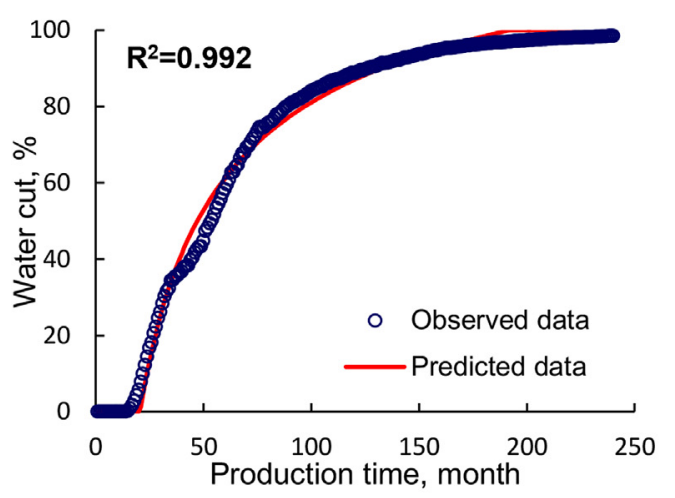

(c) Prod_3 the single time constant assumption, leading to a more reliable estimation of interwell connectivity and better agreement with the expected dynamics in synthetic waterflooding reservoir.

\subsection{Adaptation of fractional-flow equation}

In order to evaluate the adaptation of Koval fractional-flow equation, we combine the CRMIP representations with the StoSAG optimization algorithm to inversely estimate the connectivity

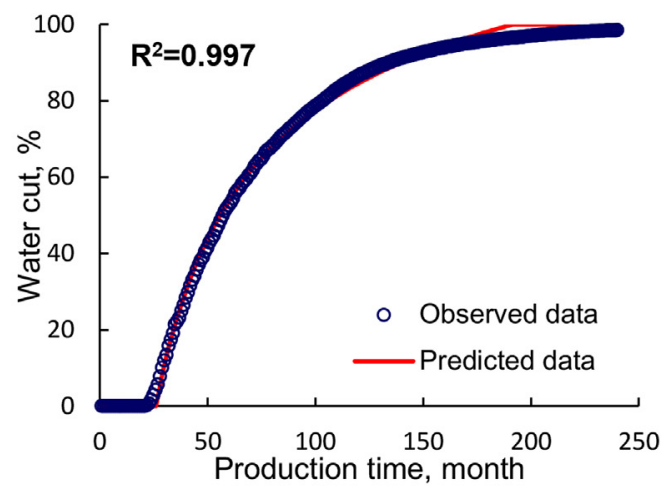

(b) Prod_2

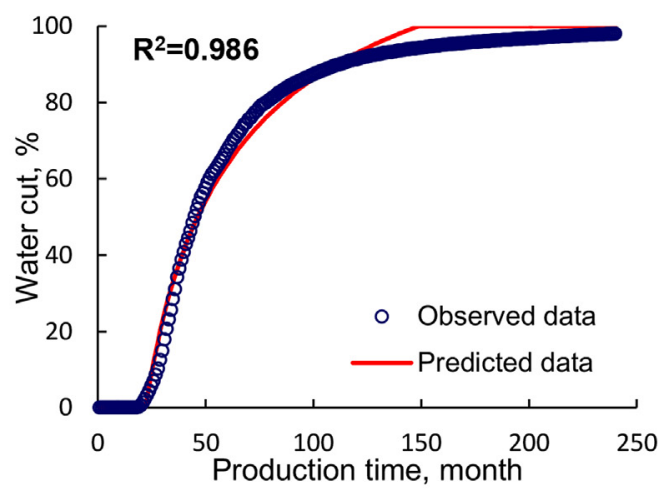

(d) Prod_4

Fig. 13. Fitting effect of the observed water cut data with respect to 4 producers. 
coefficients and time constants relevant to each injector-producer pair based control volume by history matching the observed liquid production data. On this basis, updating the Koval factor and drained pore volume sequentially, dynamic fitting of water cut data is further completed. Apart from Fig. 12(b), the other estimated geological parameters between each injector-producer pairs are presented in Table 2. Fig. 13 shows the fitting effect of the observed water cut data with respect to the 4 producers.

As can be seen, the Koval fractional flow equation is of high efficiency to describe the water-producing characteristics from small-to-large water cut period including water breakthrough. However, when waterflooding is under extra-high water cut period $\left(f_{\mathrm{w}}>90 \%\right)$, using the Koval fractional-flow equation will lead to a great deviation, which gives rise to great difficulties to a good understanding of remaining oil distribution and decision-making of potential-tapping strategies.

\section{Conclusions}

(1) The producer-based and injector-producer pair based capacitance-resistance models, CRMP and CRMIP, are respectively utilized to compute the liquid production rate of each producer associated with each reservoir control volume, followed by description of observed water cut data using the Koval fractional-flow equation. Then, a novel framework that enables the newly developed StoSAG algorithm to optimize interwell connectivity in waterflooding reservoirs by preconditioning the hybrid nonlinear constraints is finally proposed.

(2) Case study indicates that, compared to the PG and EnKF methods, the StoSAG optimization technique can handle the sequential data assimilation in large-scale nonlinear dynamics more robustly; due to more degrees of freedom, the CRMIP representation captures the reservoir's dynamic behavior better than CRMP, resulting in a more satisfactory estimation of geological parameters relative to each reservoir control volume; The Koval fractional-flow equation are effective to represent the water-producing characteristics from small-to-large water cut period, but a great deviation will be caused during the extra-high water cut stage $\left(f_{\mathrm{w}}>90 \%\right)$ because of its inherent drawbacks.

(3) In contrast to traditional reservoir simulation, the proposed ensemble-based technique can be regarded as an effective way for reservoir performance prediction and further used for real-time production optimization in stronglyheterogeneous waterflooding reservoirs, which also provides a theoretical analysis for rapid estimation of interwell connectivity by solving large-scale nonlinear optimization problems. On the other hand, great efforts should be made to the multi-layer capacitance resistance model considering the effects of cross flow between the layers and the difference of drained recoverable reserve along the vertical direction, which will be discussed in great detail later.

\section{Acknowledgements}

The authors thank PetroChina Company for the permission to publish the paper and the financial support of the PetroChina Science and Technology Major Project (Grant No. 2014E-3203), and the Important National Science and Technology Specific Projects of China (Grant No. 2016ZX05015-002).

\section{Nomenclature}

$q\left(t_{0}\right) \quad=$ liquid production rate of one producer at initial time of history matching

$I(t) \quad=$ injection rate of one injector at time $t$

$N_{\text {pro }} \quad=$ total number of producers

$N_{i n j} \quad=$ total number of injectors

$P_{w f} \quad=$ bottom-hole pressure of one producer at time $t$

$J \quad=$ productivity index of one producer

$\tau \quad=$ time constant for volume drained by one producer

$f_{i j} \quad=$ connectivity coefficient between injector $i$ and producer $j$

$c_{t} \quad=$ total compressibility

$V_{p} \quad=$ drained pore volume

$\Delta t \quad=$ time interval

$f_{w} \quad=$ water cut at the outlet of reservoir control volume

$K_{v a l}=$ Koval factor

$t_{D} \quad=$ dimensionless time

$J(\cdot) \quad=$ least-squared objective function

$\mathbf{u} \quad=$ vector of unknown controlling variables

$\mathbf{v} \quad=$ log-transformed controlling vector corresponding to $\mathbf{u}$

$u^{\text {low }} \quad=$ lower limits of the controlling variable $u$

$u_{i}^{u p} \quad=$ upper limits of the controlling variable $u$

$n_{i} \quad=$ number of inequality constraints

$n_{e} \quad=$ number of equality constraints

$A_{T} \quad=$ Jacobian matrix of inequality constraint functions

$A_{\xi} \quad=$ Jacobian matrix of equality constraint functions

C $\quad$ column vector containing the inequality constraint values

E $\quad=$ column vector containing the equality constraint values

$L_{a}(\cdot) \quad=$ augmented Lagrangian function

$\lambda=$ Lagrange multiplier

$s \quad=$ scaling factor

$\mu \quad=$ penalty parameter

$\sigma_{c_{v}} \quad=$ constraint violation factor

$n_{v} \quad=$ number of violated constraints, $n_{v}=n_{i}+n_{e}$

$\eta \quad=$ constraint-violation tolerance

a $\quad=$ inner-loop optimization iteration step size

d $\quad=$ search direction vector

$\|\cdot\|_{\infty} \quad=$ infinite norm of the controlling vector

$\xi_{f}^{l}, \xi_{u}^{l}=$ convergence criteria for the inner-loop optimization iteration

$\xi_{f}^{h}, \xi_{u}^{h}=$ convergence criteria for the outer-loop optimization

iteration $=$ convergence criteria for the outer-loop optimization iterationSubscripts and Superscripts

$i \quad=$ injector index

$j \quad=$ producer index

$k=$ time interval index in Section $2.1=$ time interval index in Section $2.1=$ inner-loop optimization iteration index in Section 3

$s \quad=$ time interval index

$e \quad=$ variable associated with equality constraints

$c \quad=$ variable associated with inequality constraints

$l \quad=$ outer-iteration index

\section{References}

Cao, F., Luo, H.S., Lake, L.W., April 2014. Development of a Fully Coupled Two-phase Flow Based Capacitance Resistance Model (CRM). Paper presented at the SPE Improved Oil Recovery Symposium, 12-16, Tulsa, Oklahoma, USA.

Cao, F., Luo, H.S., Lake, L.W., 2015. Oil-rate forecast by inferring fractional-flow models from field data with Koval method combined with the capacitanceresistance model. SPE Reserv. Eval. Eng. 18 (4), 534-553.

Chen, B.L., Reynolds, A.C., 2016. Ensemble-based optimization of the wateralternating-gas injection process. SPE J. 21 (3), 786-797.

Chen, Y., Oliver, D.S., Zhang, D., 2009. Efficient ensemble-based closed-loop production optimization. SPE J. 14 (4), 634-645. 
Chen, C., Li, G., Reynolds, A.C., 2012. Robust constrained optimization of short- and long-term net present value for closed-loop reservoir management. SPE J. 17 (3), 849-864.

Do, S.T., Reynolds, A.C., 2013. Theoretical connections between optimization algorithms based on an approximate gradient. Comput. Geosci. 17 (6), 959-973.

Fonseca, R.M., Leeuwenburgh, O., Van den Hof, P.M.J., 2014. Ensemble-based hierarchical multi-objective production optimization of smart wells. Comput. Geosci. 18 (3-4), 449-461.

Fonseca, R.M., Chen, B.L., Jansen, J.D., et al., 2016. A Stochastic Simplex Approximate Gradient (StoSAG) for Optimization under Uncertainty. Int. J. Numer. Methods Eng. http://dx.doi.org/10.1002/nme.5342.

Gao, G.H., Reynolds, A.C., 2006. An improved implementation of the LBFGS algorithm for automatic history matching. SPE J. 11 (1), 5-17.

Gentil, P.H., 2005. The Use of Multi-linear Regression Models in Patterned Waterfloods: Physical Meaning of the Regression Coefficients. The University of Texas, Austin.

Holanda, R.W., Gildin, E., Jensen, J.L., November 2015. Improved Waterflood Analysis Using the Capacitance-resistance Model within a Control Systems Framework. Paper Presented at the SPE Latin American and Caribbean Petroleum Engineering Conference, 18-20, Quito, Ecuador.

Jafroodi, N., Zhang, D.X., 2011. New method for reservoir characterization and optimization using CRM-EnOpt approach. J. Pet. Sci. Eng. 77, 155-171.

Jin, Y.X., Lin, C.Y., He, X.Y., et al., 2004. Uncertainty analysis of remaining oil predicted with reservoir numerical simulation. J. Univ. Pet., China 28 (3), 22-29.

Kang, Z.H., Chen, L., Lu, X.B., et al., 2012. Fluid dynamic connectivity of karst carbonate reservoir with fracture \& cave system in Tahe Oilfield. Earth Sci. Front. 19 (2), 110-120.

Katterbauer, K., 2015. History Matching for Steam Drive Heavy Oil Reservoirs Using Ensemble-based Techniques-A Synthetic Wafra Oilfield Case Study. Paper presented at the EUROPEC, 1-4 June held in Madrid, Spain.

Kaviani, D., Valko, P.P., Jensen, J.L., April 2010. Application of the Multiwall Productivity Index-based Method to Evaluate Interwell Connectivity. Paper Presented at the SPE Improved Oil Recovery Symposium, 24-28, Tulsa, Oklahoma, USA.

Lee, K.H., Ortega, A., Ghareloo, A., et al., 2011. An active method for characterization of flow units between injection/production wells by injection-rate design. SPE Reserv. Eval. Eng. 14 (4), 433-445.

Lerlertpakdee, P., Jafarpour, B., Gildin, E., 2014. Efficient production optimization with flow-network models. SPE J. 19 (6), 1083-1095.

Mamghaderi, A., Pourafshary, P., 2013. Waterflooding performance prediction in layered reservoirs using improved capacitance-resistive model. J. Pet. Sci. Eng. 108, 107-117.

Moreno, G.A., 2013. Multilayer capacitance-resistance model with dynamic connectivities. J. Pet. Sci. Eng. 109, 298-307.

Nguyen, A.P., 2012. Capacitance Resistance Modeling for Primary Recovery, Waterflood and Water-CO2 Flood. University of Texas at Austin, Austin, Texas. Ph.D. Dissertation.
Odi, U., Lane, R.H., Barrufet, M.A., May 2010. Ensemble Based Optimization of EOR Processes. Paper presented at the SPE Western Regional Meeting, 27-29 held in Anaheim, California, USA.

Oliveira, D.F., Reynolds, A.C., 2014. An adaptive hierarchical multiscale algorithm for estimation of optimal well controls. SPE J. 19 (5), 909-930.

Oliver, D.S., Reynolds, A.C., Liu, N., 2008. Inverse Theory for Petroleum Reservoir Characterization and History Matching. Cambridge University Press, Cambridge.

Sakazar-Bustamante, M., Gonzalez-Gomez, H., Matringe, S., et al., April 2012 Combining Decline-curve Analysis and Capacitance/resistance Models to Understand and Predict the Behavior of a Mature Naturally Fractured Carbonate Reservoir under Gas Injection. Paper presented at the SPE Latin America and Caribbean Petroleum Engineering Conference, 16-18, Anaheim C A.

Sayarpour, M., Zuluaga, E., Kabir, C.S., et al., November 2007. The use of capacitanceresistance models for rapid estimation of waterflood performance. In: Paper Presented at the SPE Annual Technical Conference and Exhibition, 11-14. California, U.S.A, Anaheim.

Su, H.J., Oliver, D.S., 2010. Smart well production optimization using an ensemblebased method. SPE Reserv. Eval. Eng. 13 (6), 1-9.

Tafti, A., Ershaghi, T., Rezapour, I., et al., April 2013. Injection Scheduling Design for Reduced Order Waterflood Modeling. Paper presented at the SPE Western Regional \& AAPG Pacific Section Meeting, 19-25 Joint Technical Conference, Monterey, CA.

Tao, Q., Bryant, S.L., 2015. Optimizing carbon sequestration with the capacitance/ resistance model. SPE J. 20 (5), 1094-1102.

Yousef, A.A., Gentil, P.H., Jensen, J.L., et al., 2006. A capacitance model to infer interwell connectivity from production- and injection-rate fluctuations. SPE Reserv. Eval. Eng. 9 (5), 630-646.

Yousef, A.A., Lake, L.W., Jensen, J.L., April 2006. Analysis and Interpretation of Interwell Connectivity from Production and Injection Rate Fluctuations Using a Capacitance Model. In: Paper Presented at the SPE/DOE Symposium on Improved Oil Recovery, 22 - 26. Tulsa, Oklahoma.

Zhang, Z.Q., Li, H., Zhang, D.X., 2015. Water flooding performance prediction by multi-layer capacitance-resistive models combined with the ensemble Kalman filter. J. Pet. Sci. Eng. 127, 1-19.

Zhao, H., Chen, C., Do, S., et al., 2013. Maximization of a dynamic quadratic interpolation model for production optimization. SPE J. 18 (6), 1012-1025.

Zhao, H., Kang, Z.J., Sun, H.T., et al., 2015. An interwell connectivity inversion model for oil-water dynamic simulation of multilayer reservoirs. Pet. Explor. Dev. 42 (6), $1-8$.

Zhao, H., Kang, Z.J., Zhang, X.S., et al., February 2015. INSIM: a Data-driven Model for History Matching and Prediction for Waterflooding Monitoring and Management with a Field Application. Paper Presented at the SPE Reservoir Simulation Symposium, 23-25, Houston, Texas, USA.

Zhao, H., Li, Y., Cui, S.Y., et al., 2016. History matching and production optimization of water flooding based on a data-driven interwell numerical simulation model. J. Nat. Gas Sci. Eng. 31, 48-66. 